\title{
Grado de conocimiento del ictus entre población de área rural en la provincia de Lleida
}

\author{
M. Oró ${ }^{\text {b }}$ J. Sanahuja-Montesinos ${ }^{\text {a }}$, L. Hernández ${ }^{\text {a }}$, E. Setó ${ }^{\text {a }}$, F. Purroy ${ }^{\text {a }}$
}

\begin{abstract}
GRADO DE CONOCIMIENTO DEL ICTUS ENTRE POBLACIÓN DE ÁREA RURAL EN LA PROVINCIA DE LLEIDA
\end{abstract}
Resumen. Introducción. Pese a que el ictus es la principal causa de discapacidad y una de las principales causas de muerte, el conocimiento de sus síntomas, sus causas y de la existencia del tratamiento fibrinolítico es escaso en la población general. Objetivo. Establecer el grado de conocimiento del ictus (terminología, factores de riesgo, síntomas y actitud) en el área rural de Lleida (Baix Segrià). Sujetos y métodos. Estudio de población mediante cuestionario cerrado realizado de forma aleatoria en tres centros de salud sobre un total de 153 sujetos. Resultados. Un 6,5\% desconoce completamente la enfermedad. El término 'ictus' sólo lo reconoce el 26,7\%, y el término 'embolia', el 86,9\%. Veinte personas reconocen más de siete términos. Hay un buen conocimiento de los factores de riesgo (más de cuatro factores de riesgo y menos de dos distractores) en sólo 25 $(16,3 \%)$ encuestados. El síntoma más reconocido es la alteración del habla (70,6\%). El conocimiento de los síntomas es óptimo (más de cuatro síntomas y menos de dos distractores) en 32 (20,9\%). El 68\%, en caso de ictus, avisaría primero al médico de cabecera. Si se tratase de un ataque isquémico transitorio, el 84,5\% acudiría primero al médico de cabecera; de ellos, el $27 \%$ no de forma urgente. Los encuestados con estudios universitarios son los que mejor conocen los factores de riesgo y los síntomas, y los que mejor actúan frente a un ictus ( $\mathrm{p}<0,05)$. Los mayores de 65 años son los que peor conocimiento tienen de la enfermedad. Conclusión. El conocimiento de la enfermedad es insatisfactorio en el área rural de Lleida. Son necesarias campañas de información a la población general para mejorar la implantación del Código Ictus en esta zona. [REV NEUROL 2009; 48: 515-9]

Palabras clave. Conocimiento de factores de riesgo. Educación sanitaria. Ictus. Síntomas de alerta.

\section{INTRODUCCIÓN}

La enfermedad cerebrovascular aguda (ECVA) o ictus es la patología neurológica aguda más frecuente y una de las principales causas de muerte, discapacidad y minusvalía en el adulto $[1,2]$. Hay suficientes evidencias que indican que la atención urgente del paciente con ictus, en un medio hospitalario bien dotado de los medios materiales y personales (neurólogos expertos) necesarios para poder aplicar un programa protocolizado de cuidados, métodos diagnósticos y tratamiento específico (unidades de ictus o en su defecto equipos de ictus), mejora significativamente la evolución de los pacientes afectados [38]. La existencia de un fármaco seguro y eficaz, el activador tisular del plasminógeno recombinante, durante las primeras tres horas de evolución del ictus isquémico agudo ha abierto un nuevo horizonte esperanzador en el tratamiento y manejo de la ECVA [9-11]. Sin embargo, son pocos los pacientes que llegan a beneficiarse del tratamiento fibrinolítico, aproximadamente el 5\% [12]. La demora en la llegada al hospital se considera la principal causa de este hecho [13-16]. Este retraso en la llegada al hospital reside, entre otros factores, en el desconocimiento de los síntomas y factores de riesgo (FR) asociados a la ECVA [4,13,15,17-21]. Diversos estudios documentan este desconocimiento en las regiones metropolitanas de nuestro país, donde casi un $10 \%$ de la población desconoce por completo la enfermedad y sólo 4 de cada 10 personas tienen un buen

\footnotetext{
Aceptado tras revisión externa: 03.02.09.

${ }^{a}$ Unidad de Ictus. Sección de Neurología. Hospital Universitari Arnau de Vilanova. Universitat de Lleida. ${ }^{b}$ Centro de Primaria Ronda. Institut Català de Salut. Fundación de Investigación de Medicina de Primaria Jordi Gol i Gurina. Lleida, España.
}

Correspondencia: Dr. Francisco Purroy. Unidad de Ictus. Sección de Neurología. Hospital Universitari Arnau de Vilanova. Universitat de Lleida. Avda. Rovira Roure, 80.E-25198 Lleida.E-mail: fpurroygarcia@gmail.com

C 2009, REVISTA DE NEUROLOGÍA conocimiento de la sintomatología y de los FR asociados a la ECVA $[18,21,22]$.

Desde inicios del año 2005 se ha instaurado el Código Ictus en la región sanitaria de Lleida. Teniendo en cuenta que un porcentaje importante de la población que abarca el área de influencia de nuestro hospital es rural [23], nos proponemos estudiar el nivel de conocimiento acerca de la ECVA y la respuesta frente a ella en el ámbito rural de la zona del Baix Segrià de la provincia de Lleida.

\section{SUJETOS Y MÉTODOS}

Se llevó a cabo un estudio de población en el que los sujetos, elegidos de forma aleatoria, contestaron un cuestionario cerrado. La encuesta se realizó en los centros de salud de las principales poblaciones que forman la región del Baix Segrià: Massalcoreig, 44; la Granja d'Escarp, 35; Serós, 15, y Aitona, 59.

\section{Encuesta}

En la encuesta se recogieron datos sobre la edad, el sexo, el nivel educativo y la existencia de un familiar o conocido próximo afecto de ictus de los sujetos que contestaron la encuesta. Además, se establecieron los siguientes apartados:

- Conocimiento de la terminología que la población general utiliza para referirse a la ECVA: embolia, feridura, infarto cerebral, accidente cerebrovascular, derrame cerebral, trombosis cerebral y ataque isquémico transitorio (AIT). De forma directa, se les interrogó sobre el conocimiento del término 'ictus'.

- Cuál es la fuente de información sobre la ECVA: médico, enfermería, neurólogo, familiar o conocido, diarios o televisión, escuela o instituto e internet.

- Reconocimiento de los FR asociados a la ECVA: mediante respuestas cerradas tipo sí o no, se enumeran seis FR vascular intercalados (hipertensión arterial, diabetes mellitus, dislipemia, tabaquismo, edad y enfermedades cardíacas) con variables distractoras (depresión, estreñimiento y fiebre).

- Identificación de signos y síntomas que suceden en el ictus: de igual modo, con respuestas cerradas se enumeraron seis signos o síntomas característicos de la ECVA (alteración del habla, dificultad para entender, déficit motor, alteración sensitiva y alteración visual) con variables distractoras (fiebre y disnea). 
- Actitud ante una situación simulada de un paciente que acaba de tener un ictus establecido y de un paciente que ha sufrido un episodio transitorio de déficit neurológico.

\section{Definiciones}

Consideramos un buen conocimiento de los FR asociados a la ECVA si reconocían más de tres FR y se confundían con menos de dos factores distractores. De igual modo, el conocimiento de más de tres síntomas característicos de la ECVA con un máximo de un error se aceptó como un conocimiento óptimo.

\section{Estudio estadístico}

El análisis estadístico de los datos se realizó con el programa SPSS v. 12.0 Para la comparación entre variables categóricas se utilizaron el test $\chi^{2}$ de Pearson y el test exacto de Fisher. La comparación de dos medias se realizó mediante la prueba $t$ de Student. Un nivel de significación $p<0,05$ se consideró estadísticamente significativo en todas las comparaciones. De igual modo, se realizó un estudio multivariante utilizando modelos de regresión logística, ajustados para las variables asociadas al buen conocimiento de FR, sintomatología y reacción frente a un ictus agudo y a un AIT.

\section{RESULTADOS}

Un total de 153 personas escogidas aleatoriamente contestaron la encuesta. Sólo $40(26,1 \%)$ eran hombres. La edad media fue de 56,1 $\pm 16,7$ años. El $49 \%$ tenía algún familiar o conocido afecto de ictus. Con respecto a su formación escolar, $107(69,9 \%)$ no tenían estudios o bien éstos eran primarios; $35(22,9 \%)$ tenían estudios secundarios, y sólo 11 (7,2\%), estudios universitarios.

El término más utilizado para referirse a la ECVA fue el de embolia $(86,9 \%)$, seguido por el de derrame cerebral $(83,7 \%)$, feridura $(82,4 \%)$, infarto cerebral $(76,5 \%)$ y trombosis cerebral $(71,9 \%)$. Un $26,8 \%$ de los sujetos reconocía la palabra 'ictus', mientras que un porcentaje muy similar identificaba el término 'accidente cerebrovascular' (27,5\%). Tan sólo cuatro personas reconocían el término 'AIT'. La media de términos conocidos fue de 4,61 $\pm 1,95$.

La principal fuente de información fue el médico de medicina familiar y comunitaria en 58 casos $(37,9 \%)$, un familiar o conocido en $50(32,7 \%)$, periódicos o televisión en $37(24,2 \%)$, internet en $17(11,1 \%)$, enfermería en $12(7,8 \%)$, un neurólogo en 9 (5,9\%); y la escuela o el instituto en $6(3,9 \%)$.

\section{Conocimiento de los FR de la ECVA}

El principal FR reconocido de la ECVA fue la hipertensión arterial, identificada en 117 casos, mientras que los otros FR fueron identificados en mucho menor porcentaje (Tabla I). Se observó un conocimiento óptimo de los FR (cuatro o más FR y menos de dos variables distractoras) en tan sólo 48 $(31,4 \%)$ sujetos. La formación universitaria del entrevistado $(p=0,086)$, que la fuente de información hubiese sido un médico de familia $(p=0,037)$, enfermería ( $p=0,036)$ o la escuela $(p=0,078)$ fueron variables asociadas al buen conocimiento de los FR. La media de términos conocidos también fue diferente entre la cohorte de entrevistados con buen o mal conocimiento de los FR $(p=0,003)$. El estudio multivariante mediante regresión logística identificó como único predictor de conocimiento adecuado de los FR de la ECVA que una fuente de información fuese enfermería -odds ratio $(\mathrm{OR})=$ 3,42 ; intervalo de confianza al 95\% (IC 95\%) $=1,03-11,38 ; p=0,046-$.

\section{Conocimiento de los signos y síntomas de la ECVA}

Los síntomas y signos de alerta de ECVA más reconocidos (Tabla II) fueron la dificultad para hablar (70,6\%), seguida del déficit motor $(66,0 \%)$. La alteración visual sólo la reconocieron el 18,3\% de los casos. Sólo 62 (40,5\%) de los entrevistados tuvieron un conocimiento óptimo de la sintomatología del ictus agudo, mientras que $88(57,5 \%)$ tuvieron un conocimiento mínimo de los síntomas de alarma de la ECVA: déficit motor y dificultad para hablar. El conocimiento de los síntomas fue óptimo (más de cuatro síntomas y menos de dos distractores) en $32(20,9 \%)$.

Las variables asociadas al conocimiento óptimo de los signos y síntomas del ictus agudo (Tabla III) fueron: el sexo mujer $(p=0,051)$, la formación universitaria $(p=0,024)$ y que una fuente de información fuese el médico de cabecera $(p=0,027)$ o un neurólogo $(p=0,1)$. Los entrevistados con mejor conocimiento fueron más jóvenes: $51,9 \pm 18,7$ frente a $59 \pm 14,5(p=$
Tabla I. Reconocimiento de los factores de riesgo asociados a la enfermedad cerebrovascular aguda.

Factores de riesgo de ictus

\begin{tabular}{ll}
\hline Enfermedades cardíacas & $54(35,3 \%)$ \\
\hline Hipertensión arterial & $117(76,5 \%)$ \\
\hline Diabetes mellitus & $60(39,2 \%)$ \\
\hline Edad & $53(34,6 \%)$ \\
\hline Dislipemia & $69(45,1 \%)$ \\
\hline Tabaquismo & $50(32,7 \%)$
\end{tabular}

Factores incorrectos

\begin{tabular}{ll}
\hline Depresión & $11(7,7 \%)$ \\
\hline Fiebre & $3(2,1 \%)$ \\
\hline Estreñimiento & $5(3,5 \%)$ \\
\hline
\end{tabular}

Tabla II. Reconocimiento de los síntomas asociados a la enfermedad cerebrovascular aguda.

Síntomas sugestivos de ictus

\begin{tabular}{lc}
\hline Dificultad para hablar-expresarse & $108(70,6 \%)$ \\
\hline Dificultad para entender & $66(43,1 \%)$ \\
\hline Déficit motor & $101(66,0 \%)$ \\
\hline Déficit sensitivo & $70(45,8 \%)$ \\
\hline Alteración visual & $28(18,3 \%)$ \\
\hline Cefalea intensa & $70(45,8 \%)$ \\
\hline
\end{tabular}

Síntomas incorrectos

\begin{tabular}{lc}
\hline Fiebre & $7(4,6 \%)$ \\
\hline Disnea & $19(12,4 \%)$
\end{tabular}

0,002). Se observó una asociación entre buen conocimiento de FR y de signos o síntomas de ECVA $(p=0,001)$.

La formación universitaria $(\mathrm{OR}=4,95 ; \mathrm{IC} 95 \%=1,23-19,96 ; p=0,024)$ y que una fuente de información hubiese sido el médico de cabecera $(\mathrm{OR}=$ $2,25$; IC $95 \%=1,13-4,46 ; p=0,021)$ se comportaron como predictores independientes de buen conocimiento de la sintomatología del ictus agudo en el estudio multivariante mediante regresión logística.

\section{Actitud frente a la ECVA y al AIT}

En el primer caso, en el que se plantea qué hacer ante un conocido o familiar que acaba de sufrir un ictus agudo, de los 153 encuestados respondieron 142 . De ellos, $12(8,4 \%)$ contestaron que esperarían a ver si se resolvía de forma espontánea la sintomatología del enfermo; 30 (21,1\%), que irían por sus medios al hospital; 14 (9,9\%) llamarían al 061; y el resto, $86(60,6 \%)$, acudirían o se pondrían en contacto con el médico de cabecera de forma urgente.

No se observó una relación directa entre el conocimiento adecuado de la clínica o los FR de la ECVA y una correcta actitud frente a un ictus agudo. Así, sólo 17 de $62(27,4 \%)$ sujetos con buen conocimiento de los síntomas y 15 de $48(31,25 \%)$ con óptimo conocimiento de los FR tuvo una buena respuesta a la ECVA. Los entrevistados con formación universitaria fueron los que mejor actuaron $(p=0,015)$. La edad media de los entrevistados que aconsejaron una valoración precoz de un sujeto con ictus agudo fue significativamente más joven $(49,6 \pm 18,1$ frente a 58,3 $\pm 15,9$ años; $p=0,005)$. Tras el estudio multivariante mediante regresión logística, la única variable 
Tabla III. Estudio univariante del conocimiento de los factores de riesgo, la sintomatología y la actitud frente al ictus agudo.

\begin{tabular}{|c|c|c|c|c|c|c|}
\hline & \multicolumn{3}{|c|}{ Conocimiento óptimo de los factores de riesgo } & \multicolumn{3}{|c|}{ Conocimiento óptimo de la sintomatología } \\
\hline & No $(n=104)$ & Sí ( $n=48)$ & $p$ & No $(n=91)$ & Sí ( $n=62)$ & $p$ \\
\hline Sexo, mujer & $76(72,4 \%)$ & $37(77,1 \%)$ & 0,539 & $62(68,1 \%)$ & $51(82,3 \%)$ & 0,051 \\
\hline \multicolumn{7}{|l|}{ Estudios } \\
\hline Sin estudios & $27(25,7 \%)$ & $11(22,9 \%)$ & 0,710 & $22(24,2 \%)$ & $16(25,8 \%)$ & 0,819 \\
\hline Primarios & $48(45,7 \%)$ & $21(43,8 \%)$ & 0,821 & $48(52,7 \%)$ & $21(33,9 \%)$ & 0,021 \\
\hline Secundarios & $25(23,8 \%)$ & $10(20,8 \%)$ & 0,684 & $18(19,8 \%)$ & $17(27,4 \%)$ & 0,269 \\
\hline Universitarios & $5(4,8 \%)$ & $6(12,5 \%)$ & 0,086 & $3(3,3 \%)$ & $8(12,9 \%)$ & 0,024 \\
\hline Familiar o conocido con ictus & $48(45,7 \%)$ & $27(56,3 \%)$ & 0,226 & $29(31,9 \%)$ & $21(33,9 \%)$ & 0,795 \\
\hline Edad (años) & $57,3 \pm 16,8$ & $53,7 \pm 16,3$ & 0,225 & $59 \pm 14,5$ & $51,9 \pm 18,7$ & 0,009 \\
\hline \multicolumn{7}{|l|}{ Fuente de información } \\
\hline Médico de cabecera & $34(32,4 \%)$ & $24(50 \%)$ & 0,037 & $8(8,8 \%)$ & $4(6,5 \%)$ & 0,027 \\
\hline Enfermería & $5(4,8 \%)$ & $7(14,6 \%)$ & 0,036 & $3(3,3 \%)$ & $6(9,7 \%)$ & 0,597 \\
\hline Neurólogo & $5(4,8 \%)$ & $4(8,3 \%)$ & 0,384 & $29(31,9 \%)$ & $21(33,9 \%)$ & 0,100 \\
\hline Familiar-conocido & $35(33,3 \%)$ & $15(31,3 \%)$ & 0,799 & $24(26,4 \%)$ & $13(21 \%)$ & 0,795 \\
\hline Prensa-televisión & $24(22,9 \%)$ & $13(27,1 \%)$ & 0,571 & $2(2,2 \%)$ & $4(6,5 \%)$ & 0,443 \\
\hline Escuela & $2(1,9 \%)$ & $4(8,3 \%)$ & 0,078 & - & - & 0,183 \\
\hline Internet & $9(8,6 \%)$ & $8(16,7 \%)$ & 0,139 & - & - & - \\
\hline Número de términos reconocidos & $4,2 \pm 2$ & $5,3 \pm 1,7$ & 0,003 & $4,2 \pm 2$ & $5,2 \pm 1,6$ & 0,002 \\
\hline Conocimiento óptimo de los síntomas & $33(31,4 \%)$ & $29(60,4 \%)$ & 0,001 & - & - & - \\
\hline Conocimiento óptimo de los factores de riesgo & - & - & - & $19(20,9 \%)$ & $29(46,8 \%)$ & 0,001 \\
\hline
\end{tabular}

que se erigió como predictor independiente de una buena actuación fue la formación universitaria ( $\mathrm{OR}=4,45 ; \mathrm{IC} 95 \%=1,23-16,09 ; p=0,023)$.

Tras plantear el caso de un sujeto con clínica neurológica transitoria sugestiva de AIT, la mayoría de los entrevistados, 87 (56,9\%), acudiría o se pondría en contacto durante el mismo día con el médico de cabecera. De los 142 sujetos que respondieron, $33(21,6 \%)$ acudirían al día siguiente a su médico de cabecera, mientras que sólo 15 (9,8\%) solicitarían valoración en urgencias de un hospital y seis $(3,9 \%)$ llamarían al 061. Sólo una persona desestimó cualquier tipo de evaluación.

El estudio univariante mostró únicamente una relación inversa estadísticamente significativa con el sexo mujer $(p=0,015)$. Dicha relación también se observó en el estudio multivariante mediante regresión logística $(\mathrm{OR}=$ $0,316 ;$ IC $95 \%=0,121-0,823 ; p=0,018)$.

Tan sólo seis $(4,2 \%)$ de las 142 personas entrevistadas que contestaron toda la encuesta tuvieron un buen conocimiento de los FR, la sintomatología y contestaron de forma adecuada a la actuación frente a un ictus agudo. En cambio, $44(31,1 \%)$ no tenían ni conocimientos óptimos de FR, ni de la clínica, ni escogieron la mejor actuación frente a la ECVA. Finalmente, el desconocimiento de la enfermedad fue total en 10 personas $(6,5 \%)$.

\section{DISCUSIÓN}

En nuestro estudio se valora el grado de conocimiento de la ECVA en el área rural de Lleida. Nuestro hospital abarca una gran zona territorial con población rural, por lo que nos interesaba indagar el conocimiento de la ECVA y la actitud frente a ella.

En la literatura anglosajona sí se ha documentado una mayor prevalencia de ECVA en áreas rurales [24-27], con mayor discapacidad por la menor accesibilidad a terapias rehabilitadoras
[28] y demora a la llegada al hospital en caso de ictus agudo [24, 29]. Igualmente, un porcentaje bajo de la población rural reconoce los principales signos de alerta de la ECVA [30]. En España, un único trabajo compara la prevalencia de ictus entre población rural y urbana, sin encontrar diferencias significativas [27], pero no hay ninguna publicación que haya estudiado específicamente el conocimiento del ictus en población rural en España.

Como en estudios previos en población española [18,22, $31]$, los términos más conocidos en nuestro estudio fueron el de 'embolia', 'derrame cerebral' y 'feridura'. El término 'ictus' es de los menos conocidos, aunque el porcentaje de sujetos que lo identifican $(26,8 \%)$ es mayor que en estudios anteriores $[18,22$, 31]. La proporción de sujetos que identificaron más del $50 \%$ de los FR relacionados con el ictus fue baja. Algo menos de uno de cada tres entrevistados tiene un conocimiento óptimo. Este dato es similar al de estudios realizados en población urbana [18,22]. El papel de enfermería en la educación sanitaria resalta en nuestro trabajo. El hecho de que la información haya sido dada por este tipo de profesionales sanitarios se comporta como único predictor independiente. La asociación con un nivel de estudios universitarios, aunque sin llegar a ser estadísticamente significativa en nuestro trabajo, también se había observado en otros previos [22]. Desafortunadamente, pocos entrevistados identificaron como FR de la ECVA las enfermedades cardíacas, la diabetes mellitus y el tabaquismo. Resultados similares o incluso peores se han detectado en Estados Unidos [32] y en Europa central [19]. 
Tabla III. Estudio univariante del conocimiento de los factores de riesgo, la sintomatología y la actitud frente al ictus agudo (cont.).

\begin{tabular}{|c|c|c|c|c|c|c|}
\hline & \multicolumn{3}{|c|}{$\begin{array}{l}\text { Actitud correcta } \\
\text { frente a un ictus agudo }\end{array}$} & \multicolumn{3}{|c|}{$\begin{array}{l}\text { Valoración urgente hospitalaria o por } 061 \\
\text { de un sujeto con ataque isquémico transitorio }\end{array}$} \\
\hline & No $(n=98)$ & Sí $(n=44)$ & $p$ & No $(n=121)$ & Sí $(n=21)$ & $p$ \\
\hline Sexo, mujer & $73(74,5 \%)$ & $32(72,7 \%)$ & 0,825 & $94(77,7 \%)$ & $11(52,4 \%)$ & 0,015 \\
\hline \multicolumn{7}{|l|}{ Estudios } \\
\hline Sin estudios & $29(29,6 \%)$ & $5(11,4 \%)$ & 0,019 & $30(24 \%)$ & $4(19,0 \%)$ & 0,569 \\
\hline Primarios & $45(45,9 \%)$ & $18(40,9 \%)$ & 0,578 & $54(44,6 \%)$ & $9(42,9 \%)$ & 0,880 \\
\hline Secundarios & $20(20,4 \%)$ & $14(31,8 \%)$ & 0,141 & $29(24 \%)$ & $5(23,8 \%)$ & 0,988 \\
\hline Universitarios & $4(4,1 \%)$ & $7(15,9 \%)$ & 0,015 & $8(6,6 \%)$ & $3(14,3 \%)$ & 0,225 \\
\hline Familiar o conocido con ictus & $49(50 \%)$ & $25(56,89$ & 0,452 & $65(53,7 \%)$ & $9(42,9 \%)$ & 0,358 \\
\hline Edad (años & $58,3 \pm 15,9$ & $49,6 \pm 18,1$ & 0,005 & $56,5 \pm 16,5$ & $50,4 \pm 19,6$ & 0,132 \\
\hline \multicolumn{7}{|l|}{ Fuente de información } \\
\hline Médico de cabecera & $38(38,8 \%)$ & $17(38,6 \%)$ & 0,987 & $48(39,7 \%)$ & $8(38,1 \%)$ & 0,892 \\
\hline Enfermería & $7(7,1 \%)$ & $4(9,1 \%)$ & 0,688 & $10(8,3 \%)$ & $1(4,8 \%)$ & 1,000 \\
\hline Neurólogo & $7(7,1 \%)$ & $1(2,3 \%)$ & 0,244 & $8(6,6 \%)$ & $0(0 \%)$ & 0,605 \\
\hline Familiar-conocido & $29(29,6 \%)$ & $16(36,4 \%)$ & 0,423 & $36(29,8 \%)$ & $8(38,1 \%)$ & 0,445 \\
\hline Prensa-televisión & $22(22,4 \%)$ & $13(29,5 \%)$ & 0,364 & $28(23,1 \%)$ & $7(33,3 \%)$ & 0,317 \\
\hline Escuela & $3(3,1 \%)$ & $3(6,8 \%)$ & 0,374 & $4(3,3 \%)$ & $2(9,5 \%)$ & 0,216 \\
\hline Internet & $13(13,3 \%)$ & $4(9,1 \%)$ & 0,479 & $13(10,7 \%)$ & $4(19 \%)$ & 0,281 \\
\hline Número de términos reconocidos & $4,5 \pm 2,1$ & $5 \pm 1,4$ & 0,142 & $4,7 \pm 1,9$ & $4,7 \pm 2,0$ & 0,909 \\
\hline Conocimiento óptimo de los síntomas & $45(45,9 \%)$ & $17(38,6 \%)$ & 0,418 & $55(45,5 \%)$ & $7(33,3 \%)$ & 0,301 \\
\hline Conocimiento óptimo de los factores de riesgo & $33(33,7 \%)$ & $15(34,1 \%)$ & 0,866 & $40(33,1 \%)$ & $7(33,3 \%)$ & 0,980 \\
\hline
\end{tabular}

El reconocimiento de los signos y síntomas de alerta se consigue aproximadamente en el $40 \%$ de los entrevistados, mientras que tres de cada cinco son capaces de identificar los principales síntomas de la ECVA, el déficit motor y la alteración del lenguaje. Nuevamente, de forma similar a los estudios realizados en nuestro país [18,22], la formación universitaria se asoció con el buen conocimiento de la sintomatología. En este caso, el médico de cabecera parece ser la mejor fuente de información.

Paradójicamente, aunque ya se ha descrito previamente [19], la buena noción de los FR y de la sintomatología no se asocia a una buena actitud frente al ictus agudo. El buen conocimiento no parece ser suficiente para mejorar la demora de la llegada al hospital [33]. En nuestro estudio, sólo una de cada tres personas con buen conocimiento de la ECVA actúa de forma adecuada ante su aparición.

A diferencia de estudios previos [18,19,22,24,28,29,31], la mayoría de los sujetos acudiría antes a su médico de cabecera ante un caso de ictus agudo que llamar al $061 \mathrm{o}$ ir directamente al hospital. Este hecho es más llamativo en el caso de un AIT, en el que casi cuatro de cada cinco entrevistados acudirían a su médico de cabecera.

Otro dato preocupante de la encuesta es que la población mayor, que es la de mayor riesgo, resulta la menos informada sobre la ECVA.
La principal limitación del estudio es el tamaño muestral. Un mayor número de participantes podría proporcionarnos más datos sobre los diferentes estamentos de edad, para saber la mejor fuente de información. Además, en nuestra cohorte hay un predominio de mujeres entrevistadas.

En conclusión, este trabajo puede ayudar a entender la idiosincrasia de la asistencia médica en nuestra área rural y a saber priorizar las campañas sobre el ictus agudo. Parece quedar bien demostrado el papel fundamental que desempeña el médico de cabecera en este tipo de medios. Las campañas deberían estar destinadas no sólo a un mejor conocimiento de los FR para prevenir la ECVA o a la sintomatología para detectar el ictus agudo, sino a extender el concepto de que el ictus es una patología con tratamiento y que éste es más eficaz cuanto antes se administra. No es suficiente con que la población reconozca cuándo sucede un ictus, sino que debe saber que existe un tratamiento efectivo y que el tiempo hasta la llegada al hospital es crucial para obtener un mejor resultado. Además, también podrían ser beneficiosas las campañas dirigidas tanto al personal de enfermería como a los facultativos de la especialidad de medicina de familia y comunitaria para consensuar la mejor manera de derivar los pacientes candidatos a tratamiento fibrinolítico al hospital de referencia. 


\section{BIBLIOGRAFÍA}

1. Feigin VL, Lawes CM, Bennett DA, Anderson CS. Stroke epidemiology: a review of population-based studies of incidence, prevalence, and case-fatality in the late 20th century. Lancet Neurol 2003; 2: 43-53.

2. Fernández de Bobadilla J, Sicras-Mainar A, Navarro-Artieda R, Planas-Comes A, Soto-Álvarez J, Sánchez-Maestre C, et al. Estimación de la prevalencia, incidencia, comorbilidades y costes directos asociados en pacientes que demandan atención por ictus en un ámbito poblacional español. Rev Neurol 2008; 46: 397-405.

3. Álvarez-Sabín J, Molina C, Montaner J, Arenillas J, Pujadas F, Huertas $\mathrm{R}$, et al. Clinical benefit following the implementation of a specialized urgent stroke care system. Med Clin (Barc) 2004; 122: 528-31.

4. Dávalos A, Castillo J, Martínez-Vila E. Delay in neurological attention and stroke outcome. Cerebrovascular Diseases Study Group of the Spanish Society of Neurology. Stroke 1995; 26: 2233-7.

5. Barreiro-Tella P, Díez-Tejedor E, Frank-García A, Lara-Lara M, Fuentes B. Organización asistencial en el cuidado agudo de ictus. Las unidades de ictus marcan la diferencia. Rev Neurol 2001; 32: 101-6.

6. Aboderin I, Venables G. Stroke management in Europe. Pan European Consensus Meeting on Stroke Management. J Intern Med 1996; 240 : 173-80.

7. Álvarez-Sabín J, Molina CA, Abilleira S, Montaner J, García-Alfranca F, Jiménez-Fábrega $X$, et al. Stroke code impact on the efficacy of thrombolytic treatment. Med Clin (Barc) 2003; 120: 47-51.

8. Álvarez-Sabín J, Alonso de Leciñana M, Gallego J, Gil-Peralta A, Casado I, Castillo J, et al. Plan for stroke healthcare delivery. Neurologia 2006; 21: 717-26.

9. The National Institute of Neurological Disorders and Stroke rt-PA Stroke Study Group. Tissue plasminogen activator for acute ischemic stroke. N Engl J Med 1995; 333: 1581-7.

10. Dávalos A, Álvarez-Sabín J, Martí-Vilalta JL, Castillo J. Intravenous tissue-type plasminogen activator for the treatment of acute cerebral ischemia. Med Clin (Barc) 2003; 120: 1-5.

11. Wahlgren N, Ahmed N, Davalos A, Ford GA, Grond M, Hacke W, et al. Thrombolysis with alteplase for acute ischaemic stroke in the Safe Implementation of Thrombolysis in Stroke-Monitoring Study (SITSMOST): an observational study. Lancet 2007; 369: 275-82.

12. Dávalos A. Thrombolysis in acute ischemic stroke: successes, failures, and new hopes. Cerebrovasc Dis 2005; 20 (Suppl 2): 135-9.

13. California Acute Stroke Pilot Registry I. Prioritizing interventions to improve rates of thrombolysis for ischemic stroke. Neurology 2005; 64: 654-9.

14. Yu RF, San José MC, Manzanilla BM, Oris MY, Gan R. Sources and reasons for delays in the care of acute stroke patients. J Neurol Sci 2002; 199: 49-54.

15. Engelstein E, Margulies J, Jeret JS. Lack of t-PA use for acute ischemic stroke in a community hospital: high incidence of exclusion criteria. Am J Emerg Med 2000; 18: 257-60.

16. Palomeras E, Fossas P, Quintana M, Monteis R, Sebastian M, Fábregas $\mathrm{C}$, et al. Emergency perception and other variables associated with ex- tra-hospital delay in stroke patients in the Maresme region (Spain). Eur J Neurol 2008; 15: 329-35.

17. Muller-Nordhorn J, Nolte CH, Rossnagel K, Jungehulsing GJ, Reich A, Roll S, et al. Knowledge about risk factors for stroke: a populationbased survey with 28,090 participants. Stroke 2006; 37: 946-50.

18. Montaner J, Mauleón A, Vidal C, Molina C, Álvarez-Sabín J. Ictus: un desconocido para la población. Rev Neurol 1998; 27: 943-7.

19. Mikulik R, Bunt L, Hrdlicka D, Dusek L, Vaclavik D, Kryza J. Calling 911 in response to stroke: a nationwide study assessing definitive individual behavior. Stroke 2008; 39: 1844-9.

20. Pancioli AM, Broderick J, Kothari R, Brott T, Tuchfarber A, Miller R, et al. Public perception of stroke warning signs and knowledge of potential risk factors. Jama 1998; 279: 1288-92.

21. Palomeras-Soler E, Fossas-Felip P, Quintana-Luque M, Monteis-Cahis R, Sebastián-Salvatierra M, Fábregas-Casarramona C, et al. Reconocimiento de los síntomas del ictus y respuesta frente a ellos en la población. Neurologia 2007; 22: 434-40.

22. Montaner J, Vidal C, Molina C, Álvarez-Sabín J. Selecting the target and the message for a stroke public education campaign: a local survey conducted by neurologists. Eur J Epidemiol 2001; 17: 581-6.

23. Jover-Sáenz A, Porcel-Pérez JM, Vives-Soto M, Rubio-Caballero M. Epidemiología de la enfermedad cerebrovascular aguda en la provincia de Lleida durante el período 1996-1997. Factores predictivos de mortalidad a corto y medio plazo. Rev Neurol 1999; 28: 941-8.

24. Joubert J, Prentice LF, Moulin T, Liaw ST, Joubert LB, Preux PM, et al. Stroke in rural areas and small communities. Stroke 2008; 39: 1920-8.

25. Adams PF, Hendershot GE, Marano MA. Current estimates from the National Health Interview Survey, 1996. Vital Health Stat 10 1999: 1-203.

26. Yiannakoulias N, Svenson LW, Hill MD, Schopflocher DP, Rowe BH, James RC, et al. Incident cerebrovascular disease in rural and urban Alberta. Cerebrovasc Dis 2004; 17: 72-8.

27. Bermejo F, Vega S, Morales JM, Díaz J, López L, Parra D, et al. Prevalence of stroke in two samples (rural and urban) of old people in Spain. A pilot door-to-door study carried out by health professionals. Neurologia 1997; 12: 157-61.

28. Casey MM, Thiede Call K, Klingner JM. Are rural residents less likely to obtain recommended preventive healthcare services? Am J Prev Med 2001; 21: 182-8.

29. Gebhardt JG, Norris TE. Acute stroke care at rural hospitals in Idaho: challenges in expediting stroke care. J Rural Health 2006; 22: 88-91.

30. Alkadry MG, Wilson C, Nicholson D. Stroke awareness among rural residents: the case of West Virginia. Soc Work Health Care 2005; 42: 73-92.

31. Segura T, Vega G, Lopez S, Rubio F, Castillo J. Public perception of stroke in Spain. Cerebrovasc Dis 2003; 16: 21-6.

32. Reeves MJ, Hogan JG, Rafferty AP. Knowledge of stroke risk factors and warning signs among Michigan adults. Neurology 2002; 59: 1288-92.

33. Lindley RI. Improving onset to needle time: knowledge is not enough. Stroke 2008; 39: 1667

\section{THE EXTENT OF KNOWLEDGE ABOUT STROKES AMONG THE POPULATION OF A RURAL AREA IN THE PROVINCE OF LLEIDA}

Summary. Introduction. Although stroke continues to be a significant cause of morbidity and mortality, the knowledge of warning signs and risk factors among general population is still insufficient. Aim. To assess the current knowledge of stroke (terminology, signs and symptoms, risk factors and attitude) among rural population of Baix Segrià in Lleida in order to the best target and message, prior to educational campaigns. Subjects and methods. A structured interview using closeended questions was conducted among 153 users of Primary Health Centers. Results. In our cohort, 6.5\% ignored the disease, while $48(31,4 \%)$ had good knowledge of risk factors (more than three risk factors) and $62(40,5 \%)$ identified more than three warnings signs correctly. Only 31,1\% would correctly act if stroke occurred. Transient ischemic attack was not considered as emergency. Most participants, $78,5 \%$, would contact to primary care. Surprisingly, knowledge of stroke symptoms and risk factors was not associated with an increased chance of calling 061 or going to the hospital. Older respondents were less likely to recognize symptoms and to consider stroke as an emergency, while the university education was associated with good knowledge and actuation. Conclusion. The level of knowledge of established stroke risk factors, warning signs, and treatment in rural population of Lleida is low. Our data suggested not only that a community-based education program to increase public knowledge of stroke among rural population is necessary, but also we need to inform the people stroke is treatable. [REV NEUROL 2009; 48: 515-9]

Key words. Health education. Risk factors knowledge. Stroke. Warning signs. 